\title{
The role of bars on bulge formation and the evolution of barred galaxies
}

\author{
I. Pérez ${ }^{1,2}$, P. Sánchez-Blázquez ${ }^{3}$ and \\ A. Zurita ${ }^{2}$ \\ ${ }^{1}$ Kapteyn Astronomical Institute, University of Groningen, The Netherlands \\ ${ }^{2}$ Dpto. de Física Teórica y del Cosmos, Universidad de Granada, Spain \\ ${ }^{3}$ Centre for astrophysics, University of Central Lancashire, UK
}

\begin{abstract}
We have started a long term project to analyse the role played by bars in bulge formation and the formation and evolution of bars from the point of view of their star formation histories and stellar populations. To this aim, we have obtained high S/N long slit spectra of 22 barred galaxies, ranging in morphological types between $\mathrm{S} 0$ to $\mathrm{Sb}$ galaxies. We have obtained Lick/IDS indices along the bar for all the sample galaxies. We used Vazdekis et al. (2007) models to derive simple stellar population ages and metallicities. Extreme care has been taken to correct the indices for any nebular contribution. Preliminary results show that the bulges of barred galaxies are similar to those of unbarred galaxies. The metalicity gradients along bars show a dependency with the central stellar velocity dispersion. The galaxies with higher central dispersion appear to have a gradient in both age and metallicity changing radially outward to a younger and more metal rich population. The galaxies with lower central velocity dispersion show a change in age and metallicity with respect to the inner part of the bar with the populations at the end of the bar being younger or same age and more metal poor. There is no correlation between the gradient and the morphological or nuclear type. There is no correlation either with the total $K$-band luminosity. The fact that we observe galaxies with the outer parts of the bar more metal rich than the inner parts shows that at least some bars are old, since it requires long time for the observed enrichment to take place. The gradients in these bars are opposite to what is generally found in disks. The correlation between the central velocity dispersion and the bar gradient relates both, the dynamical and stellar population aging of the bar .
\end{abstract}

Keywords. galaxies: evolution, galaxies: abundances, galaxies: bulges, galaxies: stellar content

\section{Introduction}

It is widely recognised the role played by bars as main mechanisms in galaxies for the transference of angular momentum and the redistribution of matter on large scales. The modelling of bars has gone a long way, however, the observational tests have not progressed much since the work by Martin \& Friedli $(1997,1999)$ on the analysis of the gas-phase abundances along bars. They showed that there is little variation in the chemical abundances in the gas phase along the bar. Studying stellar ages and metallicities provide us with archeological clues as to the formation and evolution of the bar and the underlying disk and bulge. We have already presented the evidence for gradients in the stellar indices in Pérez, Sánchez-Blázquez and Zurita (2007) in a sample of 6 barred galaxies. We present here some preliminary results derived from the study of the stellar populations of the whole sample. 


\section{Metallicity and gradients along bars}

To measure the index gradient along the bars, we chose as the inner bar region the radius where the central sigma drops to a more less constant value after the central dip, plateau or maxima. The last data points are considered the outer bar region. Only for two galaxies, after visually checking the SDSS images, the data does not reach the end of the bar. The trends on the gradiens between the inner and the outer parts of the bar a robust to the binning choice. There seems to be a dichotomy; the galaxies showing central stellar $\sigma$ values below $\approx 140 \mathrm{~km} \mathrm{~s}^{-1}$, show a systematic behaviour with younger, or similar age, and more metal poor stellar populations at the end of the bar compared to those of the inner bar. On the other hand, the outer parts of the bars of galaxies with higher central velocity dispersion, are more metal rich and younger than the inner bar. We have checked the gradients against different galaxy parameters, such as $K$-band luminosity (as obtained from the 2MASS survey), bar-strength (from different sources in the literature), nuclear type (classification from different sources in the literature). There seems to be a week dependence with the bar strength; although, this needs to be further investigated since not all the galaxies presented a value for their bar strengths published in the literature. No correlation has been found for the other parameters investigated.

\section{The bulges of barred galaxies}

Most bulges seem to have decreasing metallicity with increasing radius in agreement with the results obtained by Moorthy \& Holtzman (2006) on the stellar populations of bulges in barred and unbarred galaxies. Contrary to Moorthy \& Holtzman (2006), we do not see a trend in the bulge gradient. They noticed that where a positive gradient was found, it was always a barred galaxy. This discrepancy is probably due to a selection effect, they have 10 barred galaxies and only three of them of earlier type than SBb.

\section{Discussion}

At least some of the bars are long lasting structures, as seen from the fact that their populations at the end of the bar are more metal rich than the inner parts. This trends are opposite to those found in disks and it implies it had to be slowly enriched after the bar was formed. These old bars present higher velocity dispersions that those we consider as younger bars. This increase in the central stellar velocity dispersion is probably related to the dynamical aging of the bar.

\section{Acknowledgements}

We thank the organisers for a very enjoyable conference. I.P acknowledges financial support from the Netherlands Organisation for Scientific research (NWO) through a Veni fellowship. A.Z. acknowledges support from the Consejería de Educación y Ciencia de la Junta de Andalucía. This work was also funded by the Plan Nacional del Espacio del Ministerio de Educación y Ciencia español. This research was supported by a Marie Curie Intra-European Fellowship within the 6th European Community Framework Programme.

\section{References}

Martin, P. \& Friedli, D. 1997, A\&A 326, 449

Martin, P. \& Friedli, D. 1999, A\&A 346, 769

Moorthy, B. \& Holtzman, J. 2006, MNRAS 371, 583

Pérez, I., Sánchez-Blázquez, P., \& Zurita, A. 2007, A\&A 465, 9 


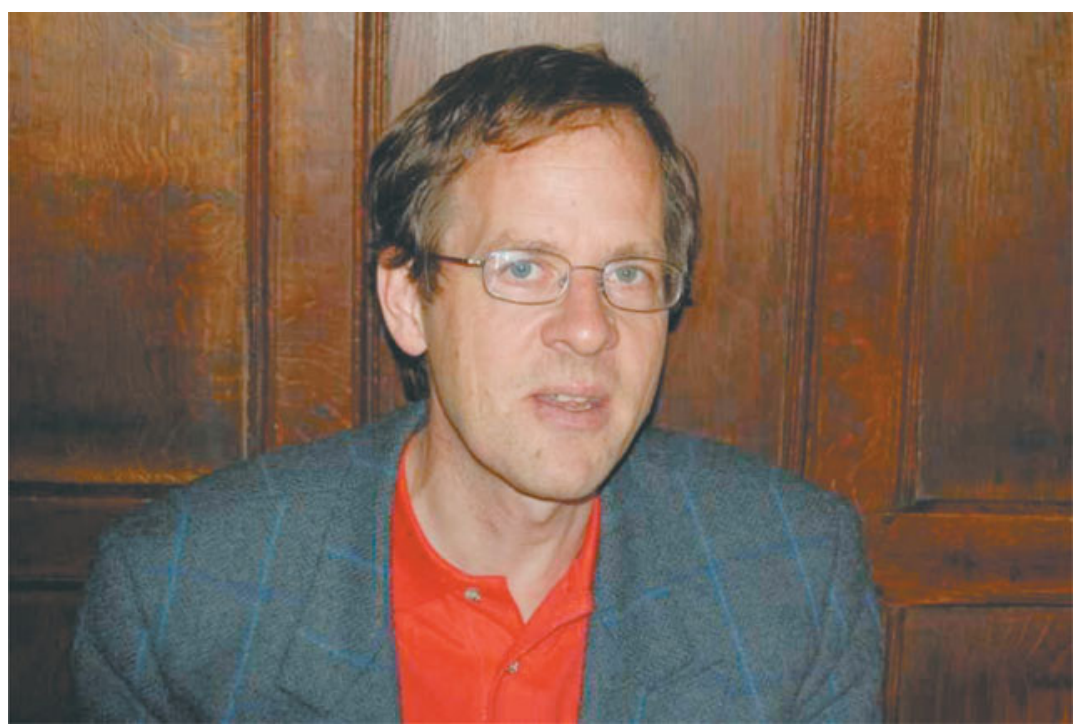

Figure 1. Reynier Peletier during the symposium dinner.

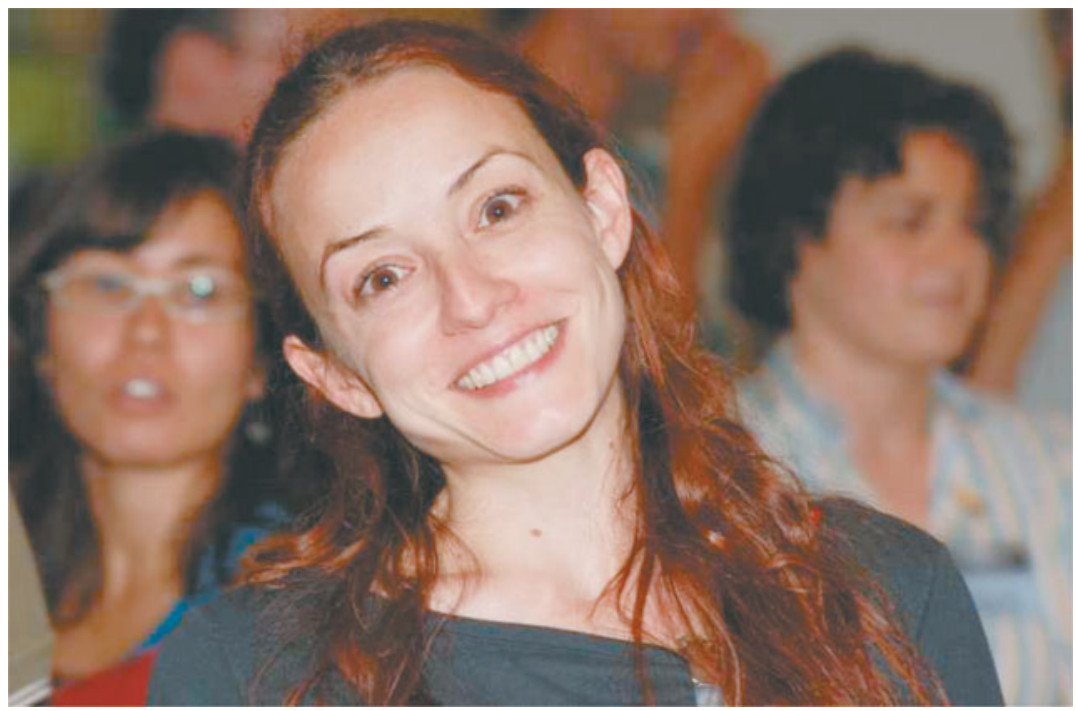

Figure 2. Patricia Sánchez-Blázquez during a coffee break. Lilian Domínguez-Palmero (left) and Genevieve Graves (right) are visible in the background. 


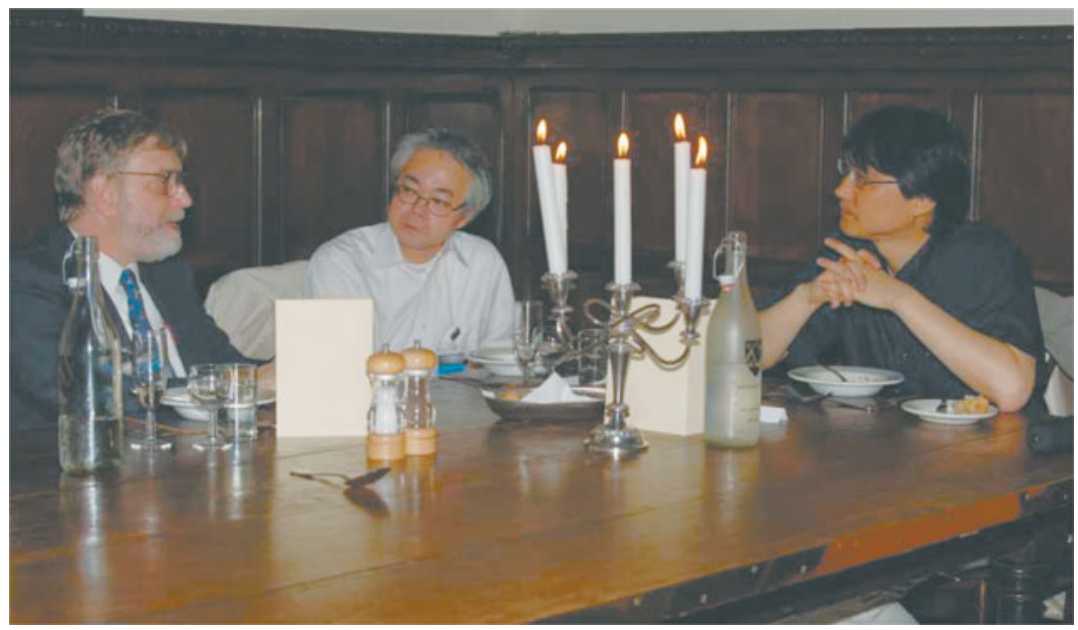

Figure 3. SOC members Douglas Richstone, Nobuo Arimoto and Sukyoung Yi at high table during the symposium dinner.

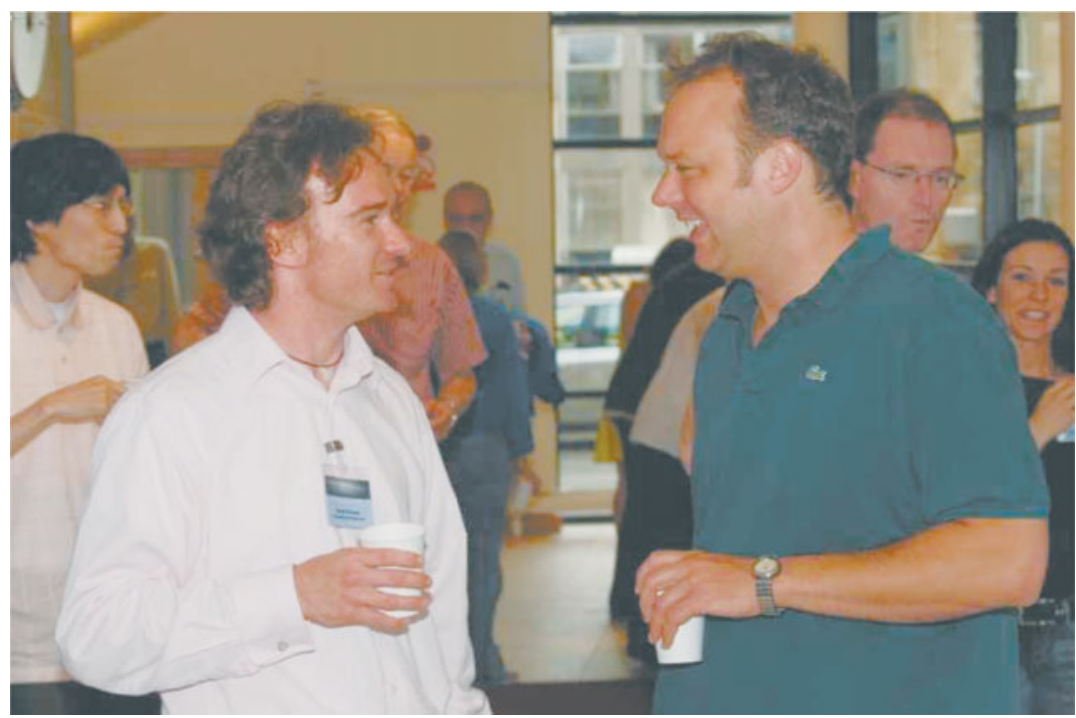

Figure 4. Daniel Thomas and Thorsten Naab discussing during a coffee break. Taihei Yano (left), James Binney (centre), John Magorrian and Alessandra Beifiori (right) are visible in the background. 


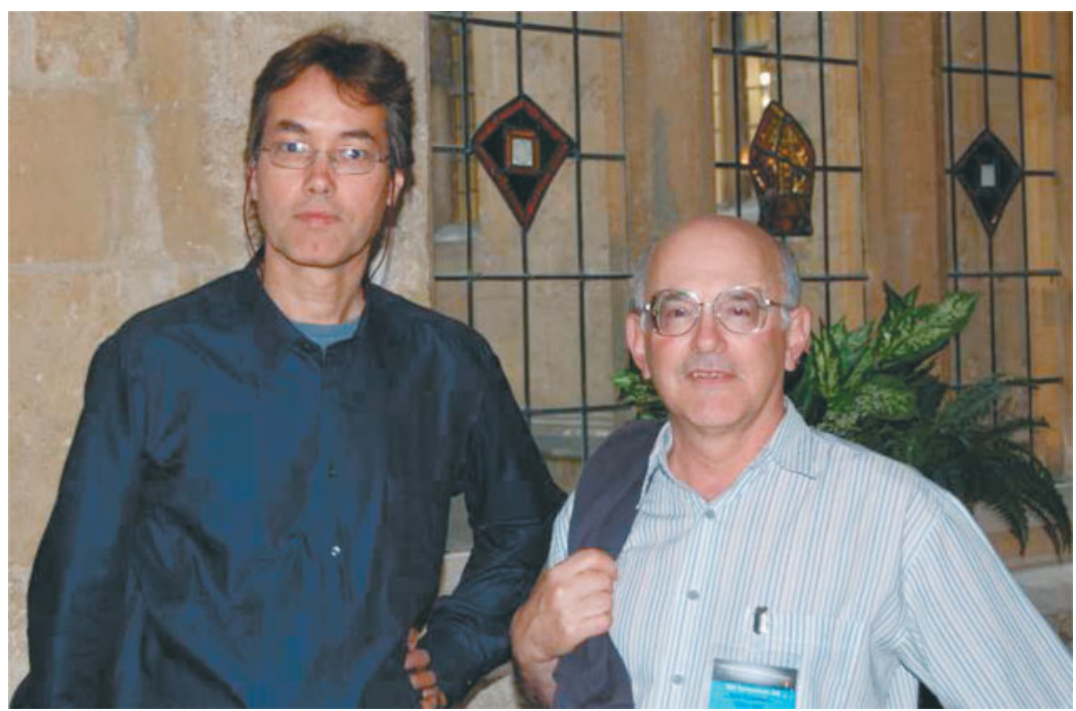

Figure 5. Marc Balcells and SOC member Ken Freeman discussing in the Wadham College cloister before the symposium dinner.

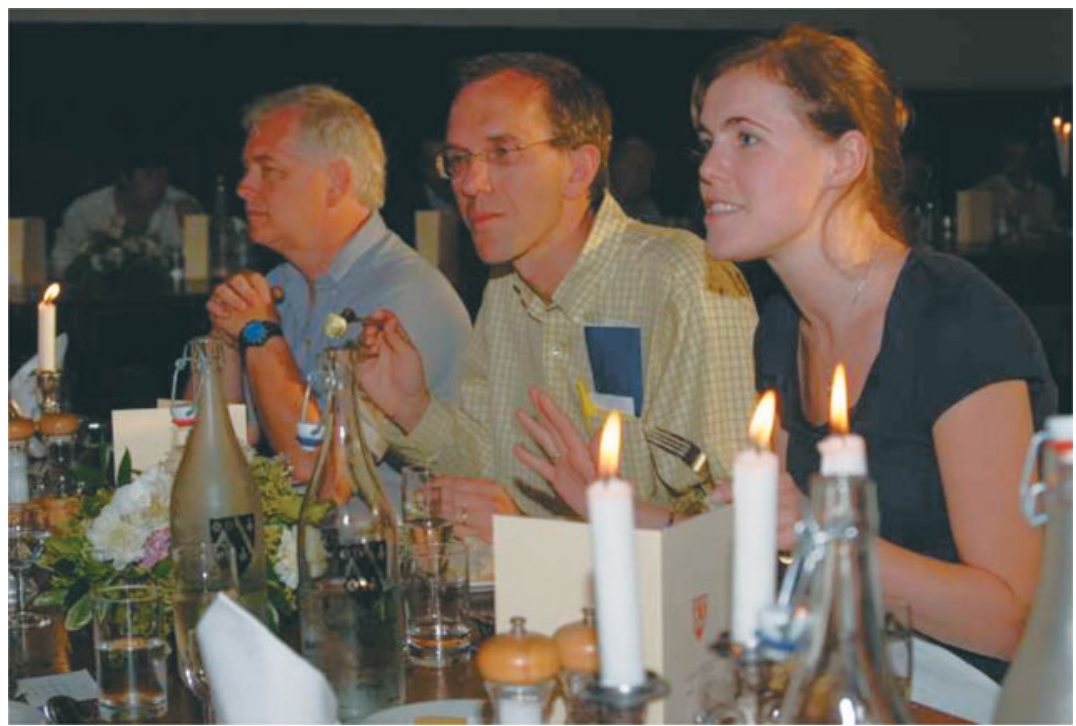

Figure 6. Christopher O'Dea, Joseph Shields and Elise Laird during the symposium dinner. 


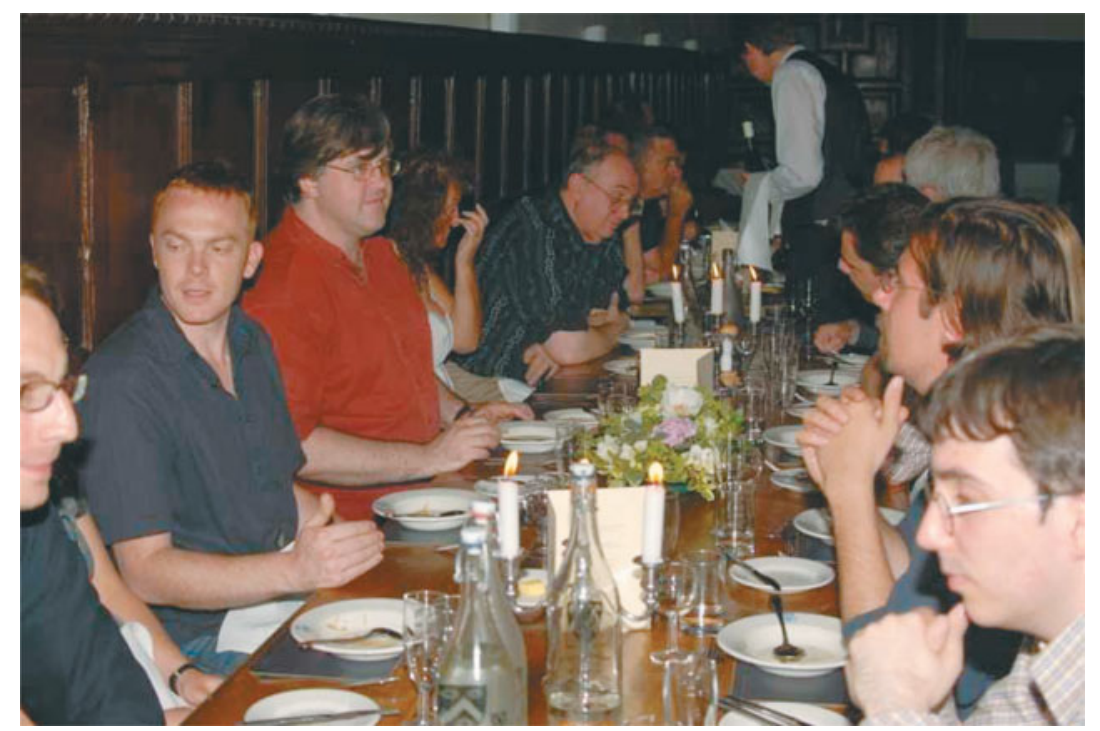

Figure 7. Flavio De Lorenzi, Maximilian Fabricius, Peter Erwin, Mary and John Kormendy (left), Giuseppe Aronica, Christian Daniel Howard, and Niv Drory (right) during the symposium dinner.

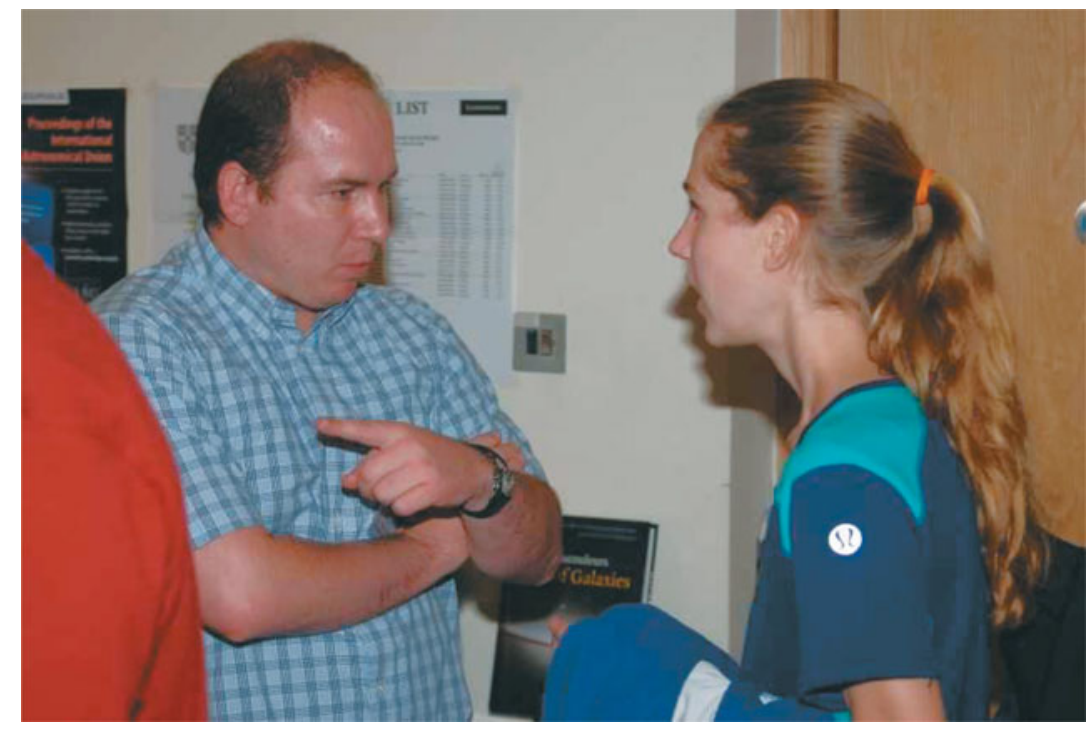

Figure 8. Jesús Falcón-Barroso and Lauren MacArthur discussing during a coffee break. 